\title{
Time transfer by laser link between China and France
}

\author{
C. Zhao ${ }^{1}{ }^{2}$ W. T. $\mathbf{N i}^{1}$ and E. Samain ${ }^{3}$ \\ ${ }^{1}$ Center for Gravitation and Cosmology, Purple Mountain Observatory, Chinese Academy of \\ Sciences, Nanjing 210008, China. \\ email: zhaocheng@pmo.ac.cn, wtni@pmo.ac.cn \\ ${ }^{2}$ Graduate University of Chinese Academy of Sciences, Beijing 100049, China. \\ ${ }^{3}$ Observatoire de la Côte d'Azur, UMR Gemini, Caussols, 06460 France. \\ email: etienne.samain@obs-azur.fr
}

\begin{abstract}
To advance from milli-arcsecond to micro-arcsecond astrometry, time keeping capability and its comparison among different stations need to be improved and enhanced. The T2L2 (Time transfer by laser link) experiment under development at OCA and CNES to be launched in 2008 on Jason-2, allows the synchronization of remote clocks on Earth. It is based on the propagation of light pulses in space which is better controlled than propagation of radio waves. In this paper, characteristics are presented for both a common view and non-common view T2L2 comparisons of clocks between China and France.
\end{abstract}

Keywords. time, standards, astrometry

\section{Introduction}

T2L2 on Jason-2 will permit to synchronize remote ground clocks and compare their frequency stabilities using laser telemetry with a performance of 1-2 orders of magnitude better than present (Samain \& Weick (2006)). T2L2 will allow to measure the stability of remote ground clocks over continental distances with 1ps over 1000s. China possesses five permanent laser ranging stations and a mobile station - TROS (Yang. (2001)), their geography allows common view comparisons among each other, and non-common view comparisons between Chinese and French stations.

\section{T2L2 principle and performance budget}

A given light pulse is emitted from station A at time $t_{s}$, and received in Jason-2 at time $t_{b}$. This pulse is also bounced from the retroreflector on Jason-2 and received at station A at time $t_{r}$. The synchronization $X_{A S}$ between the ground clock $\mathrm{A}$ and the satellite clock $\mathrm{S}$ is:

$$
X_{A S}=\frac{t_{s}+t_{r}}{2}-t_{b}+\tau_{\text {Geometry }}+\tau_{\text {Atmosphere }}+\tau_{\text {Relativity }}
$$

Also we can get $X_{B S}$, the synchronization between another ground clock B and the satellite clock. So, the time transfer between A and B can simply defined by:

$$
X_{A B}=X_{A S}-X_{B S}
$$

The performance budget is shown in Fig. 1 (Guillemot \& Samain(2006)). In the common view configuration, T2L2 should reach the majority of current atomic clocks for integration times exceeding 1000s. In non-common view, with limitations imposed by the onboard clock, T2L2 will offer an interesting alternative in calibration campaigns of radio frequency and time transfer systems based on transportable stations. 


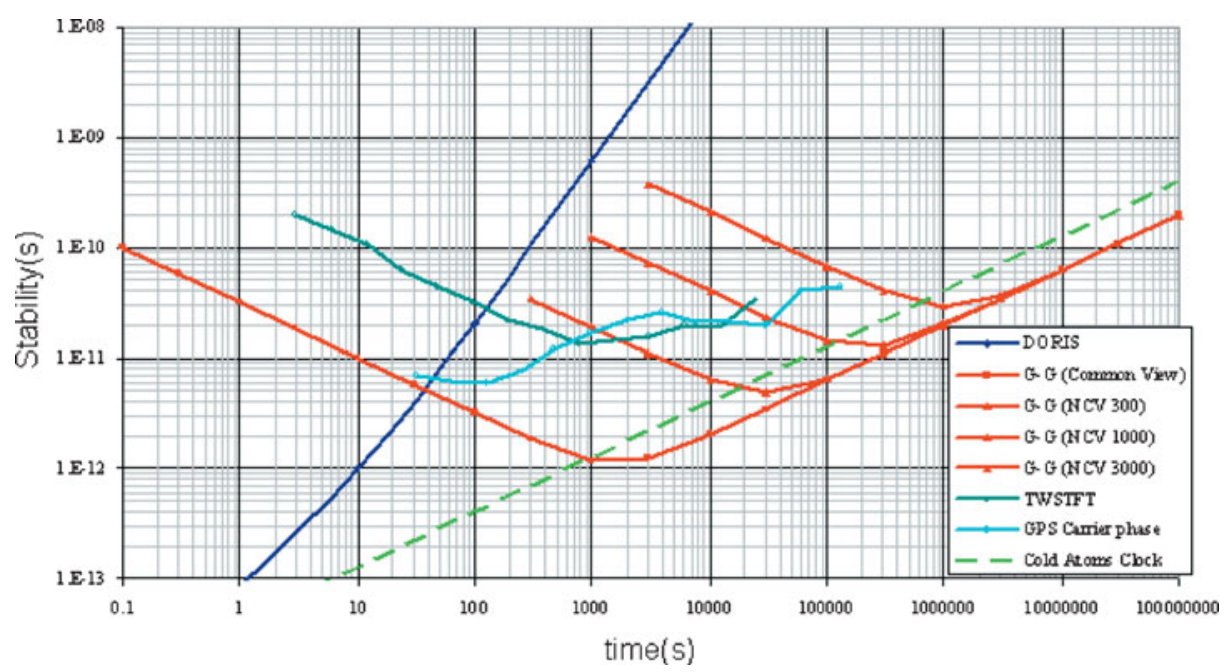

Figure 1. T2L2 ground-to-ground time stability in common view (CV) and non-common view (NCV) configuration with various interval ("NCV 300" means non-common view comparison with 300s interval between two stations).

Table 1. Grasee(France) - Chinese Stations Time Transfer in Non Common View

\begin{tabular}{l|c|c|c|ccc|ccc}
\hline Station & \multicolumn{2}{c}{ Total } & \multicolumn{2}{c}{ Good } & \multicolumn{2}{c}{ Average Duration [sec] } & \multicolumn{4}{c}{ Interval [sec] } \\
Access & Access & Grasse & China & Average & Maximum & Minimum \\
\hline Grasse-Beijing & 35 & 25 & 972.92 & 923.11 & 392.94 & 554.95 & 345.52 \\
\hline Grasse-Changchun & 32 & $\mid$ & 24 & 986.73 & 973.47 & 401.20 & 620.41 & 345.81 \\
\hline Grasse-Kunming & 26 & $\mid$ & 18 & 958.15 & 897.78 & 536.88 & 763.02 & 456.57 \\
\hline Grasse-Shanghai & 29 & $\mid$ & 20 & 1030.85 & 893.98 & 605.99 & 823.41 & 531.02 \\
\hline Grasse-Wuhan & 29 & 21 & 1021.22 & 883.47 & 560.09 & 867.04 & 489.76 \\
\hline
\end{tabular}

\section{Time transfer between Grasse and Chinese stations}

T2L2, a passenger instrument of Jason-2, will be launched by a Delta II 7320 into an earth orbit with period $6745.72 \mathrm{~s}$, inclination $66^{\circ}$ and apogee $1,336 \mathrm{~km}$ high. The orbit drifts to the east $39.5^{\circ}$ every revolution and returns to the original orbit every cycle (9.9156 days). This orbit will allow common view comparisons with about $3000 \mathrm{~km}$ baseline and six repeating ground tracks per day over the ground stations.

For common view time transfer, all five fixed laser ranging stations in China are able to track the Jason-2 synchronously with a long common duration. We expect abundant time transfer results in common view between these stations.

For non-common view time transfer, as Table 1 shows, there are around 20 good passes that each Chinese station can access Jason-2 after several minutes when Grasse station loses its sight. This is quite good for non-common view time transfer.

\section{References}

Guillemot, P. \& Samain, E. et al. 2006, in proc of the IFCS 2006., p. 771

Samain, E. \& Weick, J. et al. 2006, International Journal of Modern Physics D, in press.

Yang, F.M 2001, Surveys in Geophysics, D22, p. 465 